\title{
Reviews Reenvisioned: Supporting Enhanced Practice Improvement for Hospitalists
}

\author{
Erin Shaughnessy, MD, MSHCM ${ }^{1 *}$, Samir S Shah, MD, MSCE ${ }^{2,3}$, Read G Pierce, MD ${ }^{4}$
}

'Division of Hospital Medicine, Phoenix Children's Hospital, University of Arizona College of Medicine, Phoenix, Arizona; ${ }^{2}$ Division of Hospital Medicine, Cincinnati Children's Hospital Medical Center, Cincinnati, Ohio; ${ }^{3}$ Department of Pediatrics, University of Cincinnati College of Medicine, Cincinnati, Ohio; ${ }^{4}$ Division of Hospital Medicine, Department of Medicine, University of Colorado Anschutz Medical Campus, Denver, Colorado.

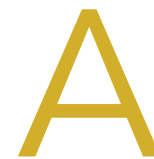

s part of the Journal of Hospital Medicine's ${ }^{\circledR}$ commitment to our readership, we are excited to announce innovative new review formats, designed for busy hospitalists. The state of knowledge in our field is changing rapidly, and the $21^{\text {st }}$ century poses a conundrum to clinicians in the form of increasingly complex studies and guidelines amidst ever-decreasing time to digest them. As a result, it can be challenging for hospitalists to access and interpret recently published research to inform their clinical practice. Because we are committed to practical innovation for hospitalists, starting in 2019, JHM will offer focused yet informative content that places important advances into relevant clinical or methodological context and provides our readers with information that is accessible, meaningful, and actionable_all in a more concise format.

Our new Clinical Guideline Highlights for the Hospitalist is a brief, targeted review of recently published clinical guidelines, distilling the major recommendations relevant to hospital medicine and placing them in context of the available evidence. This review format also offers a critique of gaps in the literature and notes areas ripe for future study. In this issue, we debut two articles using this new approach-one aimed at adult hospitalists and the other at pediatric hospitalists-regarding recently published studies and guidelines about maintenance intravenous fluids. ${ }^{1-5}$

In 2019, we will also introduce a second new format, called Progress Notes. These reviews will be shorter than JHM's traditional review format, and will accept two types of articles: clinical and methodological. The clinical Progress Notes will provide an update on the last several years of evidence related to diagnosis, treatment, risk stratification, and/or prevention of a clinical problem highly pertinent to hospitalists. The meth-

*Corresponding Author: Erin Shaughnessy, MD, MSHCM; E-mail: eshaughnessy@phoenixchildrens.com

Received: January 24, 2019; Accepted: January 24, 2019

๑) 2019 Society of Hospital Medicine DOI 10.12788/jhm.3172 odological Progress Notes will provide our readers with insight into the application of quantitative, qualitative, and quality improvement methods commonly used in work published in this journal. Our aim is to use Progress Notes as a way to enhance both clinical practice and scholarship efforts by our readers.

Finally, we will introduce "Hospital Medicine: The Year in Review," an annual feature that concisely compiles and critiques the top articles in both adult and pediatric hospital medicine over the past year. The "Year in Review" will serve as a written corollary to the popular "Updates in Hospital Medicine" presentation at the Society of Hospital Medicine annual meeting, and will highlight important research that advanced our field or provided us a fresh perspective on hospitalist practice.

As we introduce these new review formats, it is important to note that JHM will continue to accept traditional, long-form reviews on any topic relevant to hospitalists, with a preference for rigorous systematic reviews or meta-analyses. Equally important is that JHM's overarching commitment remains unchanged: support clinicians, leaders, and scholars in our field in their pursuit of delivering evidence-based, high-value clinical care. We hope you enjoy these new article formats and we look forward to your feedback.

Disclosures: The authors declare they have no conflicts of interest/competing interests.

\section{References}

1. National Clinical Guideline Centre. Intravenous Fluid Therapy: Intravenous Fluid Therapy in Adults in Hospital. London: Royal College of Physicians (UK); 2013 Dec.

2. Selmer MW, Self WH, Wanderer JP, et al. Balanced Crystalloids versus Saline in Critically III Adults, N Engl J Med. 2018 Mar 1;378(9):829-839. doi: 10.1056/ NEJMoa1711584

3. Fled LG, et. al. "Clinical Practice Guideline: Maintenance Intravenous Fluids in Children," Pediatrics. 2018 Dec;142(6). doi: 10.1542/peds.2018-3083.

4. Gottenborg E, Pierce R. Clinical Guideline Highlights for the Hospitalist: The Use of Intravenous Fluids in the Hospitalized Adult. J Hosp Med. 2019;14(3):172-173. doi: 10.12788/jhm.3178.

5. Girdwood ST, Parker MW, Shaughnessy EE. Clinical Guideline Highlights for the Hospitalist: Maintenance Intravenous Fluids in Infants and Children. J Hosp Med. 2019;14(3):170-171. doi: 10.12788/jhm.3177. 